\title{
Recognition memory for deducible information
}

\author{
RICHARD A. GRIGGS \\ University of Florida, Gainesville, Florida 32611
}

\begin{abstract}
Recent research in language comprehension indicates a constructive encoding process in which the linguistic input is transformed into some more general semantic structure which is stored. In attempting to characterize the nature of such structures, some conflicting findings have been observed for paragraphs which describe similar set-theoretic relations, linear orderings and set inclusions. While the linear ordering research supports the constructivist theory, the set inclusion work does not. The set inclusion studies, however, employed true-false tests and not the usual recognition tests. The present study investigated the processing of set inclusion paragraphs in a self-paced study/recognition test paradigm. Results supported the constructivist theory and were contrary to predictions based upon the previous set inclusion research. Subjects were unable to differentiate between presented information and unpresented deducible information. These results were discussed in terms of accuracy constraints of different tasks on memorial performance and a possible interaction of Type of Test by Universal Quantification.
\end{abstract}

Much recent research in linguistic comprehension has supported the constructivist theory of comprehension which argues that linguistic input is subjected to an abstract, constructive encoding process (e.g., Barclay, 1973; Bransford, Barclay, \& Franks, 1972; Bransford \& Franks, 1971; Cofer, 1973; Griggs, 1974a). According to the constructivist theory, when a person comprehends a paragraph of meaningful verbal material, he educes a cognitive structure for the meaning of the paragraph. He does not simply interpret and store the sentences perse, but rather transforms the linguistic input into a memory representation which is a joint function of the input information and prior knowledge.

Research by the Bransford and Franks group (e.g., Bransford, Barclay, \& Franks, 1972; Bransford \& Franks, 1972; Bransford \& Johnson, 1973; Bransford \& McCarrell, 1974) has been instrumental in popularizing the constructivist approach and inspiring other research to develop the theory. One of the main paradigms employed by the Bransford and Franks group and of most importance to the present study is a sentence memory task using a recognition test. Subjects are presented a set of sentences which permit inferences to be made. This is followed by a recognition test on presented and deducible sentences. NEWS (test sentences expressing information that could be inferred from the information in the presented sentences) are mistaken consistently for OLDS (sentences that have actually been presented). Bransford and Franks have concluded from such findings that, since subjects cannot differentiate between OLDS and NEWS, they use the input

This work was performed while the author was a graduate student at Indiana University and was supported by NIMH Grant PHS ROI MH 16817 to Frank Restle. I am deeply indebted to Dr. Restle for his continued support and assistance in this project and to Jerry C. Forshee for invaluable programming assistance. Requests for reprints should be sent to Richard $A$ Griggs, Department of Psychology, University of Florida, Gainesville, Florida 32611. sentences along with a general knowledge of the world to construct and store a general semantic description of the situation described by the input sentences and do not store verbatim copies of the sentences or merely their deep structure representations (Chomsky, 1965) The Bransford and Franks group have come up with numerous experiments to demonstrate the validity of their approach and the construction of "wholistic semantic ideas," "semantic situations," etc. But what are these ideas and situations? How can they be characterized?

Potts (1972, 1974; Scholz \& Potts, 1974) has attempted to find out exactly what is stored in this constructive encoding process for at least one type of meaningful verbal material. Potts employed English paragraphs that described transitive linear orderings which can be represented symbolically as $\mathrm{A}>\mathrm{B}>\mathrm{C}>\mathrm{D}$, where $A, B, C$, and $D$ represent the terms of the ordering and " $>$ " represents some comparative adjective. Linear orderings were used because of their transitivity, which allows information to be deduced (i.e., $A>C, B>D$, $A>D)$. The deduced pairs are referred to as remote pairs, whereas the pairs which provide the necessary information to establish the ordering (i.e., $\mathrm{A}>\mathrm{B}$, B $>$ C, C $>$ D) are called adjacent pairs.

In brief, subjects studied a paragraph, knowing they were going to be tested on the information in the paragraph, and then were given a true-false test. Test sentences were statements of the adjacent and remote pairs and their false counterparts created by reversing the terms in each pair. Unlike the Bransford and Franks studies, subjects were not required to indicate whether or not a test sentence had actually been presented. Potts found that performance (proportion correct or reaction time) on remote deducible pairs was superior to performance on adjacent presented information. This result can be accounted for by an explanation that subjects used the presented information to construct an 
ordering and stored that-not a set of the individual pairs.

Potts (1973), using his linear ordering paradigm but employing the Bransford and Franks recognition test, found more support for the argument that subjects store general semantic descriptions rather than individual sentences. Subjects mistakenly identified remote pairs as being presented. These findings provide more support for the constructivist theory and substantiate Potts' argument that subjects do alter the form of the presented ordering information and store a general semantic representation of the linear ordering.

Frase $(1969,1970,1972)$, using a similar settheoretic relation (a set inclusion instead of a linear ordering), found results inconsistent with those of Potts. In general, Frase's studies demonstrated the superiority of memory for presented information. The basic text structure of his paragraphs was a series of subset relationships among sets (a set inclusion) and can be symbolized $\mathrm{A} \rightarrow \mathrm{B} \rightarrow \mathrm{C} \rightarrow \mathrm{D} \rightarrow \mathrm{E}$, where the arrow points to the set which includes the other set. Only the adjacent pairs (e.g., All As are Bs) were presented. As with Potts' linear orderings, the stimulus material permits deducible information (i.e., $A \rightarrow C$, $B \rightarrow D, C \rightarrow E, A \rightarrow D, B \rightarrow E, A \rightarrow E)$.

Frase's experiments did, however, differ from Potts' experiments in several procedural points. Griggs $(1974 b)^{1}$ attempted to reconcile these conflicting findings by investigating both types of set-theoretic structures under comparable experimental conditions. Using a self-paced study/true-false test paradigm, Griggs (1974b) still found processing differences between the two similar structures. Potts' results for linear orderings were replicated. Performance on deducible information was superior to performance on presented information, with no significant difference between performance on true and false test statements. However, true and false differences were observed for set inclusions, and overall level of performance on set inclusions was very low compared to performance on linear orderings. When true test statements were considered, performance on presented information was superior and proportion correct was a decreasing function of step size (a measure of the spatial distance separating the two sets in the set inclusion). When false statements were considered, performance on deducible information was superior and proportion correct was an increasing function of step size. Griggs concluded that such performance was due to faulty logical processing. Subjects were committing invalid conversion errors (i.e., if told "All As are Bs," they also interpreted this to mean "All Bs are As"). Subjects also were failing to make many valid transitive inferences, and this failure was an increasing function of the distance separating the terms in the inference. Thus, subjects did not appear to integrate the input information into a more general semantic structure and store this representation as they did for linear orderings.

Griggs (1974b) found similar results for set inclusion paragraphs under several experimental conditions, even when the paragraph was in full view of the subject during testing, requiring no memory. Griggs was only able to obtain set inclusion results similar to the linear ordering results by providing prestudy instructions about the validity and invalidity of inferences in a set inclusion.

These results and Frase's findings suggest that, for paragraphs describing set inclusion structures, the information in the paragraph is not altered and changed into a general semantic structure, as in the linear ordering studies and as the information in the Bransford and Franks studies appears to be. The present experiment is designed to investigate set inclusion recognition performance using a self-paced study/test paradigm with the Bransford and Franks recognition test. If subjects do not make the transitive inferences and do not store the general set inclusion structure, then when they are required to decide if the test sentences have actually been presented, the usually observed inability on the Bransford and Franks recognition test to differentiate between OLDS and NEWS (in this case, true adjacent and remote pairs) should not be observed. Additionally, proportion correct should be higher on true adjacent pairs than on false adjacent pairs, because of the invalid conversion errors observed by Griggs (1974b). If subjects make such conversions during study, then they are likely to identify these invalid conversions as actually having been presented. Thus, the set inclusion structure, even though transitive and comparable to the linear ordering structure, could provide evidence contrary to the constructivist theory.

\section{METHOD}

Subjects

Subjects were 81 Indiana University undergraduates who participated to fulfill a course requirement. Each participated in one experimental session lasting approximately $10 \mathrm{~min}$. Twenty-seven subjects were randomly assigned to each of three experimental conditions.

\section{Apparatus}

The experiment was conducted in the Semantic Memory Laboratory at Indiana University. The experimental room consisted of eight individual booths, each booth containing a television monitor and response box. Stimuli were presented on a $30-\mathrm{cm}$ Miratel TE video monitor controlled by an ADDS (Applied Digital Data System) MRD-280 video character generator. The displays consisted of black 6 -mm-high capital letters on a white background. Responses were obtained using a twobutton response box located immediately below each video monitor. Appropriate labels for the response buttons for each question appeared on the television screen just above the buttons. Stimulus presentation and response collection were controlled by an IBM 1800 process-control computer.

\section{Materials}

The stimuli consisted of three English paragraphs. Each of the three paragraphs described a set inclusion among five sets of items. Each paragraph presented the intended set inclusion as four adjacent relations in a chained order (i.e., if $A, B, C, D$, and $E$ represent the five sets in the inclusion $A \rightarrow B \rightarrow C \rightarrow D \rightarrow E$, then the order of the adjacent pairs in the paragraph would be 
$A B, B C, C D, D E)$. These paragraphs were taken from Griggs (1974b) and are similar to those used by Frase $(1969,1970)$, except that the universal quantifier "all" was employed in each presented adjacent relation. The content of each paragraph was fictional, so any possible confounding with background knowledge was minimized. One described the natives of a foreign country, another birds of a foreign country, and the other, production of forcign cars. One of the three paragraphs is given in Table 1.

Each of the five-term inclusions used in this experiment permits testing 10 distinct relational pairs (i.e., $A B, B C, C D$, $\mathrm{DE}, \mathrm{AC}, \mathrm{BD}, \mathrm{CE}, \mathrm{AD}, \mathrm{BE}, \mathrm{AE})$. An equal number of true and false pairs were obtained by presenting the 10 pairs in both forward and reverse order, resulting in 20 test statements. For example, the false version of "All As are Bs" would be "All Bs are As."

\section{Procedure}

Only one factor was varied-content of the paragraph. There were three experimental conditions, since three paragraphs were employed. Each subject studied and was tested upon one paragraph.

One to eight subjects participated in each experimental session. All stimulus material (instructions, paragraphs, arithmetic problems, and questions) was presented on the television monitor. At the beginning of each session, the experimenter seated the subjects and familiarized them with the experimental setting. Following this, subjects were informed that they would be presented with a paragraph to read and study carefully for as long as they wished, because they would later be tested on the information in the paragraph. They were also told they would be given a short arithmetic task on which they must do well, or else their experimental results could not be used. Following these instructions, the paragraph was presented for a subjectdetermined amount of time, followed by instructions on the arithmetic task, the arithmetic task, instructions for the recognition test, and the test. Subjects were instructed to respond "Yes" to each test statement if that sentence had actually been presented and "No" if it had not been presented in the paragraph.

The purpose of the intervening arithmetic task between study. and test was to try to insure that the representation of the settheoretic information was at least stored in long-term memory and that subjects were not maintaining the information by continuous rehearsal in short-term memory. The task was simply to indicate which of two arithmetic expressions was the larger. One expression appeared on the left side of the television screen and the other on the right side. Five pairs of expressions were used. For each pair of expressions, one of the expressions had to be calculated by adding two two-digit numbers (e.g., $28+24$ ), while the other expression was just a two-digit number and required no calculation. The subject was instructed to push one of two buttons labeled "Left" and "Right" to indicate which arithmetic expression was larger, the one on the left or the one on the right. The results of this task were monitored to insure that subjects were performing the task conscientiously.

\section{RESULTS}

The same general pattern of results was observed for all three groups. In what follows, therefore, only performance averaged over the three groups of subjects will be presented.

Statistical analyses were performed using two-tailed sign tests. The proportions correct on adjacent and remote pairs as a function of the truth value of the test sentence are presented in Table 2. A "No" answer was
Table 1

A Sample Paragraph Describing a Five-Term Set Inclusion Relation

All the Fundalas are outcasts from other tribes in Central Ugala. These people are isolated from the other tribes because it is the custom in this country to get rid of certain types of people. All the outcasts of Central Ugala are hill people. The hills provide a most accommodating place to live. All the hill people of Central Ugala are farmers. The upper highlands provide excellent soil for cultivation. All the farmers of this country are peace loving, which is reflected in their art work. Altogether, there are about 15 different tribes in this area.

correct to all false sentences and to true remote sentences. A "Yes" answer was correct for adjacent true sentences only.

Proportions correct on true adjacent and false adjacent sentences were not significantly different $(z<1)$, but proportions correct for true remote and false remote sentences were $(z=6.8, p<.001)$. For true sentences, proportion correct for adjacent sentences was significantly higher than proportion correct for remote sentences $(z=6.9, p<.001)$. The opposite was found for false sentences. Proportion correct was significantly higher on remote sentences than on adjacent sentences $(z=4.8, p<.001)$. The interaction of Type of Pair by Truth Value of the test sentence was significant $(z=5.6$, $\mathrm{p}<.001$ ).

Since the remote pairs were not presented, a simple response bias for saying "Yes" might account for the low proportion correct on true remote sentences. However, such a response bias should have also affected the proportions correct for false adjacent and remote sentences since the correct answer to these was also "No." As can be seen in Table 2, no such effects were observed.

\section{DISCUSSION}

The predictions based on previous set inclusion research were not confirmed. Instead, the same pattern of results was observed that Potts (1973) observed for linear ordering paragraphs in which only adjacent pairs were presented, except that all his proportions correct were slightly higher. The proportion correct for false sentences was higher than that for true sentences, and most of this difference was due to differences on the remote pairs. Subjects were very inaccurate in deciding

Table 2

Proportions Correct on Adjacent and Remote Pairs as a Function of the Truth Value of the Sentence

\begin{tabular}{ccc} 
Pairs Tested & $\begin{array}{c}\text { True } \\
\text { Test Sentences }\end{array}$ & $\begin{array}{c}\text { False } \\
\text { Test Sentences }\end{array}$ \\
\hline Adjacent & $.738(.028)$ & $.741(.029)^{*}$ \\
Remote & $.329(.035)^{*}$ & $.897(.023)^{*}$ \\
\hline
\end{tabular}

Note-Standard errors are given in parentheses.

"The correct answer is "No, that sentence was not presented." 
whether true remote sentences had actually been presented but very accurate in their decisions on false remote sentences. Subjects found it very difficult to decide between information that was actually presented and information that had not been presented but was deducible from presented information. Guessing probability in this study was .5 ; the proportion correct on true deducible test sentences (.329) was thus less than chance. These results are consistent with the Bransford and Franks constructivist theory and Potts' research, but inconsistent with the predictions based upon earlier set inclusion work.

Griggs (1974b) found that subjects failed to identify many valid transitive inferences as true, suggesting these inferences were not being made during study and not stored in memory. Thus, it had been predicted that subjects would not identify such inferences as having been presented if they had not been made during study and then incorporated into the memory representation for the set inclusion. Subjects did, however, identify such true remote sentences as having actually been presented. Another inconsistency with the earlier set inclusion findings is that no significant difference was observed between true and false adjacent sentences. This had been predicted because of the invalid conversion errors which had been observed in Griggs (1974b).

The present data combined with the previous set inclusion results are also somewhat in conflict with the results of Brockway, Chmielewski, and Cofer (1974). Brockway et al., using similar set inclusion paragraphs but without the universal quantifiers, found that, as the accuracy set was relaxed by instructions, more items distant from the paragraph (inferences) were accepted. One set of instructions (the accuracy set) required the subject to be very accurate in deciding that each test sentence had actually been presented in the paragraph. A less strict instructional set (the inferential set) required subjects to respond yes to a sentence if it could be inferred from the paragraph. The accuracy set seems comparable to the instructions used in the present experiment, and the inferential set can be compared to the instructions used in the earlier set inclusion work. However, subjects tended to accept inferential material in the present study but not in the earlier set inclusion work.

One possible explanation is that the results of the present study reflect an atmosphere effect created by the repetitive use of the universal quantifier "all" in the paragraphs and in the test statements (Chapman \& Chapman, 1959; Woodworth \& Sells, 1935). The word "all" supposedly suggests a universal affirmative atmosphere and statements are accepted because of this general atmosphere. This does not seem very viable, since performance on the false test statements also would have been affected by the "all" atmosphere, and they were not.

A better explanation for the above disagreement in accuracy constraint also involves the use of universal quantifiers. An interaction of Type of Test (or accuracy set) by Universal Quantification may be the cause for the disagreement. Statements such as "As are Bs" may be more plausible than statements of the form "All As are Bs" and, hence, more likely to be accepted. Thus, the finding in previous set inclusion research that subjects failed to identify valid transitive inferences as true and that this failure was an increasing function of the distance separating the terms in the inference may have been at least partially due to plausibility. In natural language, the universal quantifier "all" is usually used with the definite article, e.g., "all the books." The defirite article, in turn, denotes restriction of the universe of discourse to an immediate and specific realm. Because of this restriction on the universe, sub. jects may be very uneasy about drawing inferences from long chains of such statements, because it is so easy for the universe to shift appreciably during such a chain. The word "all" may have caused subjects to become overly cautious about the truth or falsity of a remote test sentence and thus to reject remote sentences even though they were valid. Subjects might have accepted the inferences as true if the universal quantifiers had not been employed. The above described results of Brockway et al. (1974) lend some support to this hypothesis.

When the test is concerned with truth or falsity, the universal quantifier may be very important and subjects may become very cautious. When the test is concerned only with actual occurrence, the quantifier may not interfere. Experiments are currently underway investigating this possibility. Some preliminary recall results reveal that, when attempting to recall set inclusion information, subjects show a tendency to recall set inclusion statements without the universal quantifiers. This finding thus supports an argument that subjects hesitate to use the word "all," which agrees with the above interaction hypothesis.

The present results emphasize the need for careful and thorough research in this area. Under some experimental conditions, inaccurate performance on stored information can be obtained. Thus, if a researcher is trying to build a model of the memory representation that is used, he must be very careful to consider all the factors that affect memorial performance in such situations. As pointed out by Brockway et al. (1974), memory performance depends upon several factors, including what the subject is asked to do, what kind of material he has studied, the time interval between the experiment and the test, and the conditions of the test. All these factors and others, and their possible interactions, could affect the outcome of a particular test. In trying to specify a model of the cognitive structure involved, one must take all these factors into account. This is not easy. As Brockway et al. (1974) state, "the house of memory has many mansions. The problem is to devise methods which can display their structure" (p. 194). 


\section{REFERENCES}

BARCLAY, J. R. The role of comprehension in remembering sentences. Cognitive Psychology, 1973, 4, 229-254.

Bransford. J. D.. Barclay, J. R., \& Franks, J. J. Sentence memory: A constructive versus interpretive approach. Cognitive Psychology, 1972, 3, 193-209.

Bransford, J. D., \& Franks, J. J. The abstraction of linguistic ideas. Cognitive Psychology, 1971, 2, 331-350.

Bransford, J. D., \& Franks, J. J. The abstraction of linguistic ideas: A review. Cognition: International Journal of Cognitive Psychology, 1972, 1, 211-249.

Bransford, J. D., \& Johnson, M. K. Considerations of some problems of comprehension. In W. Chase (Ed.), Visual information processing. New York: Academic Press, 1973.

Bransford, J. D., \& McCARrell, N. S. A sketch of a cognitive approach to comprehension: Some thoughts about understanding what it means to comprehend. In $W$ Weimer \& D. Palermo (Eds.), Cognition and the symbolic processes. Washington, D.C: Winston-Wiley, 1974

Brockway, J., ChMielewski, D., \& Cofer, C. N Remembering prose: Productivity and accuracy constraints in recognition memory. Journal of Verbal Learning and Verbal Behavior, 1974, 13, 194-208.

Chapman, L. J., \& Chapman, J. P. Atmosphere effect re-examined. Joumal of Experimental Psychology. 1959. 58, 220-226.

Сномsкy, N. Aspects of the theory of syntax. Cambridge: M.I.T. Press, 1965.

Cofer. C. N. Constructive processes in memory. American Scientist, 1973, 61, 537-543.

Frase, L. T. Structural analysis of the knowledge that results from thinking about text. Journal of Educational Psychology Monograph Supplement, 1969, 60(6, Part 2).

FRASE, L. T. Influence of sentence order and amount of higher level text processing upon reproductivc and productive memory. American Educational Research Journal, 1970. 7. 307-319.
Frase, L. T. Maintenance and control in the acquisition of knowledge from written materials. In R. O. Freedle \& J. B Carroll (Eds.), Language comprehension and the acquisition of knowledge. Washington, D.C: Winston-Wiley, 1972.

GRIGGS, R. A. The recall of linguistic ideas. Journal of Experimental Psychology, 1974, 103, 807-809. (a)

GrigGs, R. A. Logical errors in comprehending set inclusion relations in meaningful text (Doctoral dissertation, Indiana University, 1974). Dissertation Abstracts International, 1975, 35B, 5155B. (University Microfilms No. 75-8942, 103). (b)

Potts, G. R. Information processing strategies used in the encoding of linear orderings. Joumal of Verbal Learning and Verbal Behavior, 1972, 11, 727-740.

Potrs, G. R. Memory for redundant information. Memory \& Cognition, 1973, 1, 467-470.

PotTs. G. R. Storing and retrieving information about ordered relationships. Journal of Experimental Psychology, 1974, 103. $431-439$

Scholz, K. W., \& Potтs, G. R. Cognitive processing of linear orderings. Journal of Experimental Psychology, 1974, 102. 323.326.

Woodworth, R. S., \& Sells, S. B. An atmosphere effect in formal syllogistic reasoning. Journal of Experimental Psychology, 1935, 18, 451-460.

\section{NOTE}

1. A version of Griggs (1974b) is also available as Indiana Mathematical Psychology Report No. 74-7 from the Cognitive Institute, Department of Psychology, Indiana University, Bloomington, Indiana 47401 .

(Received for publication December 22, 1975 revision accepted March 19, 1976. ) 